\title{
Análise dos softwares gratuitos para tomografia computadorizada de feixe cônico de interesse aos cirurgiões-dentistas
}

\author{
Analysis of free software for computed tomograp hy cone beam of interest to dentists
}

\section{Ana Márcia Viana Wanzeler}

Especialista em Radiologia e Imaginologia Odontológica pela FO/UFRJ

\section{Resumo}

O objetivo deste artigo foi avaliar programas de imagens odontológicas disponiveis gratuitamente na internet como ferramentas para prática do cirurgião-dentista e que funcionam em computadores pessoais. Foi realizada uma pesquisa no Medline entre os anos de 2005 a 2014, com enfoque em software utilizado para visualizar imagens tomográficas na Odontologia. Para a pesquisa dos softwares foi realizado busca no site Google e em sites especializados por programas gratuitos disponíveis para windows. Foram utilizados as palavras chaves "free dicom viewer" e "dental software". De acordo com os critérios de inclusão e exclusão três softwares foram analisados. Conhecer a existência de softwares gratuitos e que dão um grande suporte para os profissionais é de extrema importância para a Odontologia atual.

Palavras-chave: sistemas de informação em radiologia; DICOM; Odontologia assistida por computador; visualizadores de imagens médicas.

\section{Abstract}

The aim of this study was to review the dental imaging programs available for free on the Internet as tools to practice DDS and running on personal computers. A survey was conducted in Medline from 2005 to 2014, with focus on the software used to view tomographic images in dentistry. A research about the software was done at Google search site and specialized sites for free programs available for Windows. Key words were used "free dicom viewer" and "dental software." According to the criteria of inclusion and exclusion 3 softwares were analyzed. Knowing the existence of free softwares which are able to support professionals improve their jobs is a matter of great importance for the current dentistry.

Keywords: information systems in radiology; DICOM; computer-assisted dentistry; medical images viewer.

\section{Introdução}

O crescente uso de da tomografia computadorizada (CBCT) requer mudanças em nossos métodos de diagnóstico e plano de tratamento, bem como treinamento adicional (1). O padrão para imagens de tomografia computadorizada digitais é chamado de imagem digital e comunicações em medicina (DICOM). Atualmente, existe uma grande variedade de fabricantes e fornecedores de sistemas digitais (hardware e software) necessários para a avaliação das imagens obtidas de forma digital (2). No entanto, com a criação de plataformas informáticas cada vez mais desenvolvidas, surge a necessidade de interoperabilidade (3). Segundo FARMAN (4), a interoperabilidade de imagens digitais é desejável para garantir a preservação e disponibilidade da informação diagnóstica adquirida do paciente através de várias gerações de hardware e software de imagem, entre sistemas provenientes de diferentes vendedores e provenientes ou destinados a centros de referência externos. Assim, a interoperabilidade acaba por constituir um fator de extrema importância quando se conjetura a aquisição de equipamentos/ sistemas de imagem digital, pelo que é essencial que a capacidade de exportar e ler ficheiros de imagem esteja em conformidade com as normas ISO (International Organization for Standardization), sendo de referência o padrão DICOM -Digital Imaging and Communications in Medicine (1-3).

O objetivo deste artigo é pesquisar e testar diversos programas disponíveis gratuitamente para download que oferecem ferramentas para uso na área Odontológica, avaliar a viabilidade do uso em computadores pessoais e não cabe nesta pesquisa realizar propaganda nem valorizar nenhum programa em especial.

\section{Material e Método}

Foi realizado um levantamento em bases de pesquisa Medline entre os anos de 2005 a 2014, com enfoque em software utilizado para visualizar imagens tomográficas na Odontologia. Para a pesquisa dos softwares foi realizado busca no site Google e em sites especializados por programas gratuitos disponíveis para Windows. Foram utilizados as palavras chaves "free dicom viewer" e "Dental software".

Aproximadamente 75 softwares foram encontrados com o objetivo de visualizar imagens tomográficas gratuitamente, porém como critério de exclusão, foram excluídos da amostra softwares que não disponibilização ferramentas voltadas para a radiologia odontológica. Como critério de inclusão, o programa obrigatoriamente está disponível para download gratuitamente, possui ferramentas específicas para Odontologia e pode rodar em computadores pessoais e ambiente windows. Alguns programas disponibilizam o download gratuito, porém, para abrir a imagem no sistema, há um custo com chaves especificas. Os programas que estão de acordo com os critérios de inclusão e exclusão são: InVivoDentalDemo, DentalSlice e CS 3D Imaging Software.

Os softwares ImplantView e DentalView possuem versões com download gratuito, mas para abrir uma imagem tomográfica há necessidade de uma chave de acesso. Os demais programas, como, por exemplo, Orralis Dicom 
Viewer, OsiriX estão disponíveis gratuitamente, porém não possuem ferramentas voltadas para a aplicabilidade odontológica.

Para avaliar a possibilidade do uso dos softwares para Odontologia foi testado em um computador pessoal com as seguintes configurações básicas: processador 2.26 GHZ Intel Core I3, RAM 4GB com sistema operacional Microsoft Windows 7.

Foram avaliadas as funções e ferramentas que são essenciais na prática diária do cirurgião-dentista radiologista, assim como para outras especialidades na área odontológica: criar cortes panorâmicos, transversais, axiais, sagitais, coronais e para-sagital; reconstrução multiplanares (MPR); modelo de reconstrução 3D; identificação do canal mandibular no modelo 2D e 3D; simulação da posição do implante nos planos 2D e 3D; aplicação de medições de ângulos e distâncias; gerar em CD com imagens DICOM; linhas de referencias; tornar dos dados dos exames anônimos e exportar imagens em JPG, BMP e DICOM.

\section{Resultados}

Os resultados dos softwares avaliados de acordo com as especificações descritas na metodologia estão expostos na tabela I. Em seguida serão abordadas as características encontradas em cada programa avaliado.

Tabela I. Resultados das funções e programas avaliados

\begin{tabular}{|c|c|c|c|}
\hline & DentalSlice & $\begin{array}{c}\text { CS Imaging Software } \\
2.2\end{array}$ & $\begin{array}{c}\text { InVivoDental } \\
5.0\end{array}$ \\
\hline MPR & Sim & Sim & Sim \\
\hline Curva dental ajustável & Sim & Sim & Sim \\
\hline Tornar exame anônimo & Sim & Sim & Sim \\
\hline Linhas de Referências & Sim & Sim & Sim \\
\hline Reconstrução 3D & Sim & Sim & Sim \\
\hline Simulação de implante & Sim & Sim & Sim \\
\hline Medição de ângulos e distância & Sim & Sim & Sim \\
\hline Gerar em CD & Sim & Sim & Sim \\
\hline Criar cortes & Sim & Sim & Sim \\
\hline Identificar o canal mandibular & Sim & Sim & Sim \\
\hline Exportar Imagem & Sim & Sim & Sim \\
\hline DICOM & Não & Sim & Sim \\
\hline
\end{tabular}

\section{- DentalSlice}

É um software para visualização de imagens tomográficas muito utilizado por cirurgiões-dentistas para planejamento de implantes, porém há necessidade de importação das imagens originais do tomógrafo e sua transformação em um ambiente virtual para que as imagens possam ser analisadas. Com as imagens em DICOM convertidas em um arquivo DentalSlice, o programa tem como ferramentas a possibilidade de simulação de implantes; visualização em MPR; visualização dos cortes transversais e panorâmico; reconstrução em 3D; demarcar o canal mandibular, editar a curva panorâmica; medição angulares e distâncias; linhas de referências, tornar dados anônimos; gravar em CD. O programa encontra-se disponível pelo fornecedor Bioparts tamanho do arquivo $1.4 \mathrm{~KB}$, para download gratuito, porém para transformar o arquivo DICOM para Dentalslice há necessidade de um credenciamento para realizar a conversão, gerando por tanto um custo. O programa encontra-se para download http://www.dentalslice.com.br/08_downloads.php

\section{- CS 3D Imaging Software}

Trabalha com imagens de TC formato DICOM e possibilita a geração de laudos em tamanho real (1:1). Tem como características de acordo com o fornecedor Kodak 3DImagingSoftware: reconstrução de imagens panorâmicas, axiais, sagitais, coronais, para-sagitais; reconstrução em 3D; medições de ângulos e distâncias; numeração e indicação de cortes para-axais sobre as imagens axial e panorâmica; reconstrução multiplanares (MPR); linhas de referências; tonar dados anônimos; simulação de implante; marcação do canal mandibular, possibilidade de gravar em CD.

$\mathrm{O}$ tamanho do arquivo é de 2,36 KB e é fornecido pela KODAK, mas pode-se encontrar em sites para download gratuito http://kodak-dental-imaging-software-installati.software.informer.com/

\section{- InVivoDental}

É um software de imagem volumétrica projetado especificamente para os dentistas. O programa lê imagens no formato DICOM, as especificações fornecidas pelo fornecedor anatomage são: demarcação do canal mandibular; visualização em cortes panorâmico, axial, sagital, coronal e para-sagital; reconstrução em 3D; medições de ângulo e distância; possibilidade 
de gravar CD; reconstrução multiplanares (MPR); planejamento virtual de implantes; linhas de referencias; tornar dos dados dos exames anônimos e exportar imagens em JPG, BMP e DICOM. A versão testada foi a 5.0 com tamanho de 1.5 MB. O programa encontra-se para download no site http://invivodental.software.informer.com/5.0/

\section{Discussão}

Com a modernização dos sistemas de radiologia tornando as imagens digitais ficou mais prático o armazenamento, leitura da imagem e planejamento do tratamento do paciente. Com o abandono de filmes radiográficos deu-se início a utilização de computadores e notebooks, que através de softwares específicos, conseguem realizar a leitura da imagem em formato DICOM. Em decorrência do avanço da informatização e a busca em novos conhecimentos, o profissional moderno não está mais restrito a clínicas ou consultórios, os computadores portáteis acompanham esses profissionais em viagens, congressos e para suas residências. Observando esse fato uma série de empresas começaram a criar softwares com inúmeras ferramentas cada vez mais modernas que suprem a necessidade dos especialistas que utilizam desses recursos. Porém, uma grande parte desses programas necessita de chave handware e geralmente os valores são cobrados por números de máquinas operantes.

Para alguns fornecedores o conceito Free software, isto é, a versão disponível para download, é completamente funcional e pode ser instalada sem nenhum custo adicional para o profissional.

De acordo com a tabela I, os três softwares avaliados apresentam as ferramentas analisadas nesta pesquisa e que são necessárias não somente para prática diária de cirurgiões-dentistas, mas também podem ser utilizados para pesquisas científicas. Na literatura possuem muitas pesquisas que utilizam esses programas para avaliação de exames de imagens, onde através destes o pesquisador não precisa ter uma clínica radiológica com programas restritos a chaves de acesso para desenvolver uma pesquisa que envolva tomografias computadorizadas de feixe cônico. Somente o programa DentalSlice que não identifica imagens em formato DICOM. Neste caso, o profissional deverá solicitar à clínica radiológica que encaminhe o CD com as imagens convertidas em formato DentalSlice, mas, para isto, a clínica radiológica necessariamente deve ser credenciada com a empresa Bioparts, fornecedora do programa, gerando um custo a clínica.

Cada programa possui características específicas para cada ferramenta, mas todos possuem fácil manuseio, porém há necessidade de um treinamento para que possa usufruir de todos os ícones. Ao determinar o corte em curva, automaticamente gera uma reconstrução panorâmica, na qual, a espessura pode ser determinada pelo operador. Nesta imagem a delimitação do canal mandibular e o planejamento de implantes, como, por exemplo, na figura 1.

As reconstruções multiplanares (MPR) são apresentações das três visões ortogonais (axial, sagital e coronal) em três janelas separadas. É o método padronizado para visualização de dados em 3D ou o método de escolha para avaliações iniciais das imagens, uma vez que, a área de interesse pode ser visualizada em três planos, com definição e precisão, como é mostrado na figura 2. Já as reconstruções de um modelo 3D são sobreposições dos cortes ortogonais esquematizando da forma mais real a área de interesse, servindo como guias de ilustrações para cirurgias, como, por exemplo, na figura 3.

As funções de mensurações angulares e lineares são de extrema importância para demarcar uma área, extensão de uma lesão, até mesmo mensurar a altura de um rebordo alveolar. Na prática odontológica, essas ferramentas são muito utilizadas. Deixar anônimo o exame é fundamental para expor as imagens em conferências, aulas, palestras preservando os dados dos pacientes (figura 4). A possibilidade de exportar as imagens, gerar CD nos dá dinâmica e reprodutibilidade dos exames em outras máquinas.

Os três softwares mostram-se eficientes de acordo com os requisitos analisados nesta pesquisa, cada um com suas especificidades, porém esses softwares para fins de diagnósticos são controversos, uma vez que a Agência Nacional de Vigilância Sanitária (Anvisa) não possui uma regulamentação específica.
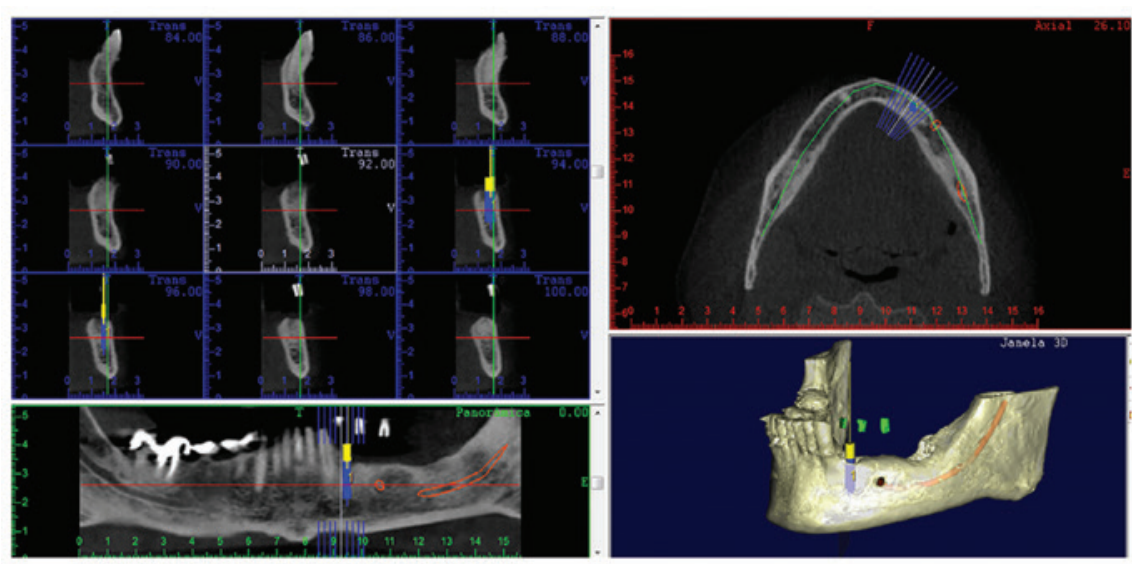

Figura 1. No programa DentalSlice delimitação do corte em curva, gerando uma reconstrução panorâmica, na qual pode-se definir a espessura e demarcar no canal mandibular. A simulação do planejamento do implante ocorre tanto na reconstrução panorâmica, como nos cortes paraxiais e ilustração no modelo 3D em uma única janela 


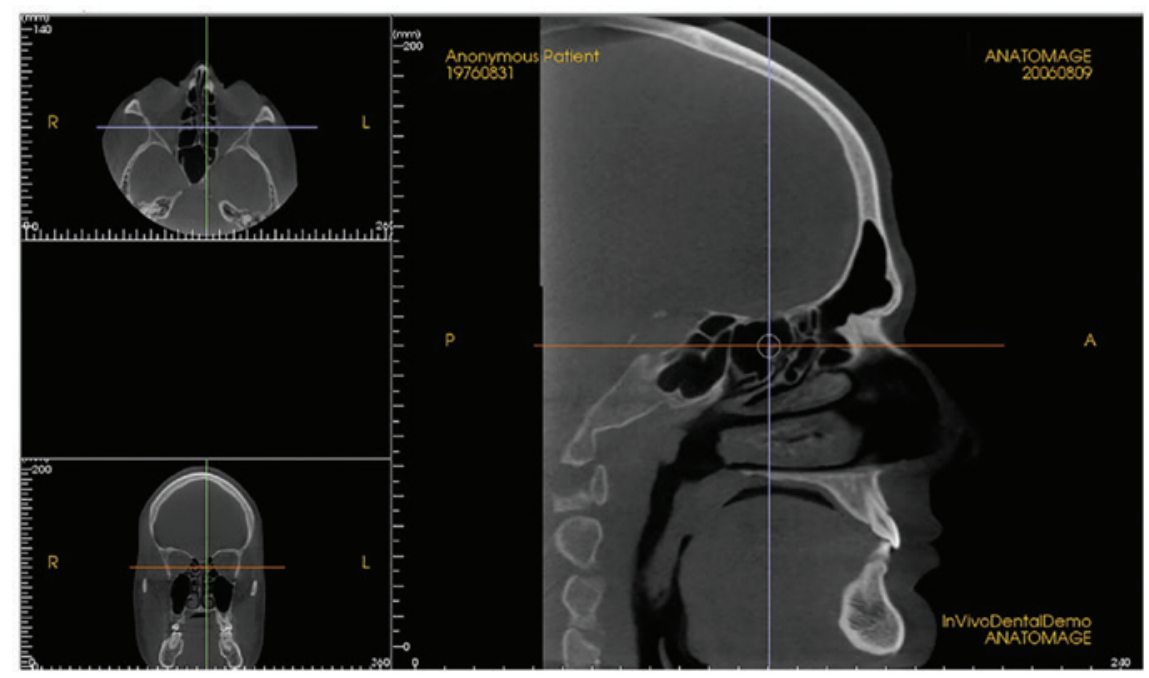

Figura 2. A função reconstrução multiplanar no programa InVivoDental: cortes sagital, coronal e axial em uma única tela

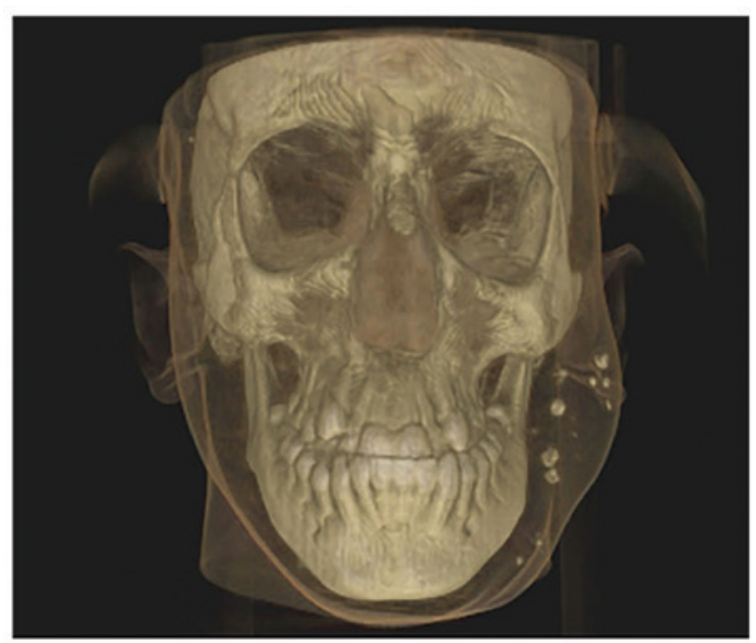

Figura 3. Ilustração em 3D do programa 3S 3D Imaging

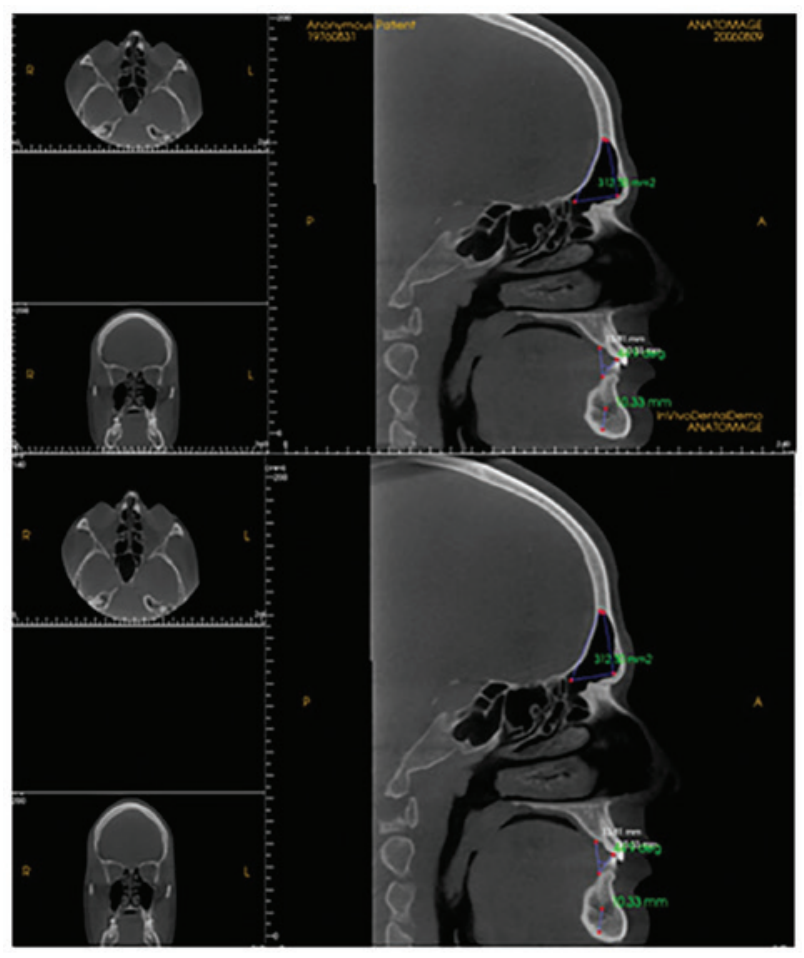

Figura 4. No programa InVivoDental a demonstração da opção de deixar o exame anônimo, remoção das linhas de orientação e possibilidades de mensurações angulares e lineares

\section{Conclusão}

Diversos são os softwares disponíveis gratuitamente com a finalidade de serem visualizadores DICOM e que dão um grande suporte para os profissionais com ferramentas necessárias, não somente para as práticas diárias dos cirurgiões-dentistas, mas também como suporte para pesquisas científicas. Nessa pesquisa os programas avaliados tiveram resultados satisfatórios e disponibilizam as ferramentas necessárias para o profissional trabalhar. Testar e avaliar esses softwares são de grande importância para que todos tenham conhecimento, lembrando que o ideal é analisar quais são as suas necessidades e então escolher o melhor programa que se adapta, sendo que se recomenda testar mais de um. 


\section{Referências ::}

1. MOREIRA, A, DURÃO, AR, CORREIA, A. Aplicação da norma DICOM em Medicina Dentária. Rev Port Estomatol Med Dent Cir Maxilofac. 2012;53(2):117-22.

2. DUARTE, HEM, ARAÚJO, CCM. Digital systems in Dentomaxillofacial Radiology: new era in Dentistry? RBO 2008; 65(1):42-7.

3. FARMAN, AG. Use and implication of the DICOM standard in dentistry. Dent Clin N Am 2002;46:565-73.

4. FARMAN, AG. Raising standards: digital interoperability and DICOM. Oral Surg Oral Med Oral Pathol Oral Radiol Endod 2005; 99: 525-6.

5. BARRA, FR, BARRA, RR, SOBRINHO, AB. Freeware medical image viewers: can we rely only on them? Radiol Bras 2010;43(5): 313-8.

6. NOBRE, LF, WANGENHEIM, AV. Free software: an option for radiologists? Radiol Bras 2010;43(5): IX-X.

7. RUBIO SERRANO, M, ALBALAT ESTELA, S, PENARROCHA DIA-
GO, M, et al. Software applied to oral implantology: update. Med Oral Patol Oral Cir Bucal 2008; 13: E661-5.

8. GOTFREDSEN, E, WENZEL, A. Integration of multiple direct digital imaging sources in a picture archiving and communication system (PACS). Dentomaxillofac. Radiol 2003;32:337-42.

9. AL-RAWI, W, JACOBS, R, HASSAN, B, et al. Evaluation of web-based instruction for anatomical interpretation in maxillofacial cone beam computed tomography, Dentomaxillofac Radiol 2007;36(8): 459-64.

10. JUNG, RE, et al. Computer Technology Applications in Surgical Implant Dentistry: A systematic review. The International Journal of Oral \& Maxillofacial Implants. 2009;24:92-109.

11. HASSAN, B, JACOBS, R, SCARFE, W, et al. A web-based instruction module for interpretation of craniofacial cone beam CT anatomy. Dentomaxillofac. Radiol 2007;36(6):348-55.

Recebido em: 21/07/2014 / Aprovado em: 22/08/2014

Ana Márcia Viana Wanzeler

Avenida Governador Magalhães Barata, 1050, apto. 501-A

Belém-PA, Brasil - CEP: 66060-281

E-mail: marciawanzeler@hotmail.com 